\title{
A Cross-layer Design Scheme between Spectrum Decision and Routing in Cognitive Wireless Mesh Networks
}

\author{
Guo-an Zhang, Yun Yang, Yan-cheng Ji \\ School of Electronics and Information \\ Nantong University \\ Nantong, China \\ Email: gzhang@ntu.edu.cn, yangyun88@hotmail.com, jiyancheng@ntu.edu.cn
}

\begin{abstract}
Spectrum sharing technologies can achieve the maximum usage of spectrum resources flexibly and highefficiently, which relieves the current spectrum crunch situation availably. In a multi-hop cognitive wireless mesh network scenario coexisting with a TDMA/FDMA cellular network, an effective scheme of cross-layer design between link-layer spectrum decision and network-layer routing is proposed, on the basis of the combination of spectrum underlay and spectrum overlay. Simulation results verify that the scheme outperforms distinctly the shortest path based random spectrum decision algorithm on network end-to-end performance.
\end{abstract}

Keywords-cognitive wireless mesh network; cross-layer; spectrum decision; routing

\section{INTRODUCTION}

As a novel broadband wireless communication system, wireless mesh network (WMN) has stand out from WLAN, Ad hoc and many other networks due to its incomparable advantages, and become an inevitable development trend of next-generation wireless technology. WMNs operate on the unlicensed bands [1]. However, with rapid development of radio communications towards broadband and multimedia, new services and new techniques are constantly emerging, which no doubt causes congest in unlicensed bands and then performance degradation of WMNs. The contradiction between supply and demand for spectrum resources hinders the continuous development of WMNs. In the meanwhile, FCC has pointed out the huge waste of the licensed spectrum, a very low utilization nearly $2 \%-6 \%$ at any time. So it is not really the lack of spectrum but the serious unbalance of spectrum usage caused by the fixed allocation policy that incurs spectrum scarcity problem. To avoid the traffic overload in unlicensed bands and simultaneously relieve the problem of spectrum crunch, there is a cry for a technology to reuse the enabled spectrum.

Hierarchical spectrum sharing access technologies can enhance the spectrum utilization through flexible spectrum access and sharing schemes, which can solve radically the aforementioned question, including spectrum underlay based on interference temperature model and spectrum overlay based on cognitive radio [2]. The concept of interference temperature and interference temperature model was proposed in 2003 by FCC [3]. Cognitive radio (CR) is an intelligent radio, which can track the variation of spectrum with time and then adjust the emitter parameters adaptively according to current environment. The technology impels the development of spectrum sharing [4].

Cognitive wireless mesh network (CogWMN) is a multihop wireless communication system where network nodes with CR function deployed in mesh structure, open spectrum access and efficient spectrum utilization for future wireless networks [5]. The CR-enabled mesh nodes (i.e. cognitive users or secondary users, SUs) can not only use the unlicensed spectrum but also access the idle bands of licensed spectrum through detecting the behavior of licensed users (i.e. primary users, PUs). Thanks to the join of CR, the performance and the throughput of WMNs are improved [6]. But the opportunistic access of license-exempt users to spectrum holes (or available channels) must rely on the high-efficient spectrum management. In order to increase spectrum utilization and guarantee the QoS of applications, SUs must be clearly aware of the available spectrum fluctuation (spectrum sensing and spectrum analysis), select the most suitable channel for their transmission (spectrum decision) and evacuate immediately once the licensees appear (spectrum handoff). Spectrum sensing, analysis, decision and handoff are all belongs to the scope of spectrum management [7]. The analyzed sensing results can provide necessary basis for decision, and handoff is mainly to ensure transparent transmission of SUs. Currently, the studies on spectrum sensing and handoff inside and outside the country have been relatively mature, while spectrum analysis and decision are still in their infancy. The purpose of spectrum decision is to fulfill the requirement of cognitive users maximally, and to realize reasonable use of spectrum resources, which is a key technology of efficient spectrum management [8].

In a CogWMN scenario coexisting with a TDMA/FDMA cellular network, SUs use the detected spectrum holes in overlay mode and sharing these channels with other SUs in underlay mode. Because of the traffic fluctuation in cellular networks, available channels are time-varying. Consequently, several spectrum handoffs will occur during the time when SUs transmit over the link. Multiple available channels and frequent spectrum handoffs make spectrum decision and routing selection more difficult. Therefore, to achieve rational usage of spectrum resources and enhance network performance, a cross-layer strategy between spectrum decision and routing is presented. 


\section{SYTEM MODEL}

Considering a CogWMN (i.e. cognitive system) composed of $n$ nodes coexisting with a TDMA/FDMA cellular network (i.e. primary system, such as GSM/EDGE radio access network called GERAN in [9]). Cognitive mesh nodes use spectrum holes of the cellular network intelligently. Suppose each cell has several pre-assigned channels and each node in the node-set Node $=\{m \mid m=1, \cdots, N\}$ of CogWMN senses the licensed channels respectively and exchanges the sensing results with others. So the number of licensed channels $\boldsymbol{C}$ equals to the channel numbers of the cellular network.

For simplicity, some important notations are listed in table I following.

TABLE I. SOME IMPORTANT NOTATIONS

\begin{tabular}{|c|c|}
\hline Notations & Description \\
\hline$m$ & a cognitive mesh node, $\quad m \in$ Node \\
\hline$T R_{m}$ & set of mesh nodes within the transmission range of $m$ \\
\hline$I R_{m}$ & set of mesh nodes within the interference range of $m$ \\
\hline$L T_{m}[c]$ & local interference temperature of channel $c$ measured at $m$ \\
\hline$L T_{m}$ & $\begin{array}{l}\forall c \in C \text {, local interference temperature vector of channel } c \\
\text { measured at } m\end{array}$ \\
\hline$P t_{m}\left(f_{c}, B_{c}\right)$ & transmission power of $m$ on channel $c$ \\
\hline$T_{m}^{m}[c]$ & $\begin{array}{l}\text { aggregate interference temperature of channel c measured at } \\
\text { the neighbors of } m \text { due to its transmissions }\end{array}$ \\
\hline$T_{m}^{n}[c]$ & $\begin{array}{l}\text { aggregate interference temperature of channel c measured at } \\
n\left(n \in \boldsymbol{I}_{\boldsymbol{m}}\right) \text { due to transmissions of } m\end{array}$ \\
\hline$L_{m n}^{c}$ & path loss in transmission from $m$ to $n\left(n \in \boldsymbol{I}_{m}\right)$ on channel c \\
\hline$T H_{c}$ & pre-defined interference temperature threshold of channel $c$ \\
\hline$k$ & Boltzmann constant, $1.38 \times 10^{-23} \mathrm{~J} / \mathrm{K}$ \\
\hline$P C_{m}$ & set of probable channels of $m$ \\
\hline$A C_{m}$ & $\begin{array}{l}\text { set of available channels of } m \text { under interference temperature } \\
\text { model }\end{array}$ \\
\hline
\end{tabular}

\section{PeR-HOP SPECTRUM DECISION AlgORITHMS}

\section{A. Set of Available Channels under Interference Temperature Model}

Interference temperature is defined as a measurement of the effective RF power delivered from receiving antenna to the receiver. It can be calculated as:

$$
T_{c}\left(f_{c}, B_{c}\right)=P_{i}\left(f_{c}, B_{c}\right) / k B_{c}
$$

Under interference temperature model, a sufficient condition for a channel $c$ to be available at node $m$ is that the interference temperature of any node within the interference transmission of node $m$ caused by its transmission on channel $c$ is less than the interference temperature threshold of the channel. The above condition can be formally represented as:

$$
T_{c}\left(f_{c}, B_{c}\right)+L_{m n}^{c} P t_{m}\left(f_{c}, B_{c}\right) / k B_{c}<T H_{c}
$$

Thus, the available channels of each mesh node are usually different.

The premise that a pair of neighbor nodes $i$ and $j$ can communicate with each other is that there is at least one channel between their separate set of available channels, i.e. $A C_{i} \cap A C_{j} \neq \varnothing$. So, in order to realize the per-hop spectrum decision algorithm, calculating the two sets $A C_{i}$ and $A C_{j}$ stands at the beginning of it all. Assuming the transmission power to be constant, the main steps of the algorithm are as follows:

1) Network initialization;

2) $\forall m \in$ Node , at each node $n \in \boldsymbol{I}_{m}$, calculate $\boldsymbol{L} \boldsymbol{T}_{n}$ and transmit $\left(n, \boldsymbol{L} \boldsymbol{T}_{n}\right)$ to all nodes within its interference range;

3) $\forall m \in$ Node , receive $\left(n, \boldsymbol{L} \boldsymbol{T}_{n}\right), n \in \boldsymbol{I R}_{\boldsymbol{m}}$ and calculate $\boldsymbol{P} \boldsymbol{C}_{\boldsymbol{m}}$ and $A C_{m}$ using following algorithms i and ii respectively.

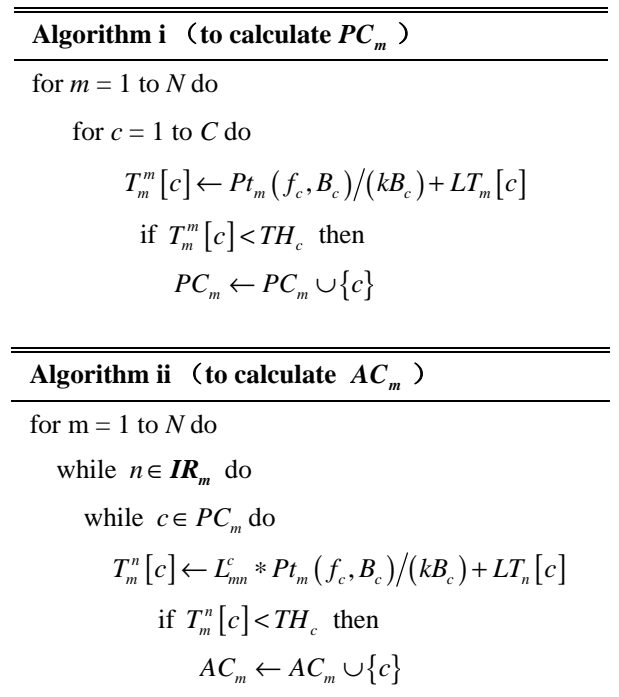

\section{B. Metric for Per-hop Channel Decision}

Spectrum decision is actually the optimization problem based on spectrum analysis. For optimal per-hop channel selection, spectrum characterization analysis is important after obtaining the available channels. Moreover, it is an effective way to protect PUs from interferences caused by the opportunistic transmission of SUs through predicting the prior information of each channel. It is thus clear that spectrum analysis is extremely necessary.

Suppose that the number of PUs in a cell is $P$ and the arrival processes of their services are Poisson. There are $Q$ channels allocated to the cell. Each channel can only be occupied by one primary user at the same time. The average arrival rate and the average service time of PUs services on different channels are $\lambda_{p}^{(q)}$ and $\mu_{p}^{(q)}$ separately, $p=1, \cdots, P$ and $q=1, \cdots, Q$.The arrival processes of the services of cognitive mesh nodes are also Poisson and their average arrival rate is $\lambda_{s}$ and if the service is not interrupted by PUs the average service time is $\mu_{s}$. SUs services are assumed to be data traffics not sensitive to latency. Therefore, PRP (Preemptive Resume Priority) M/G/1 queuing theory can be used to model the behaviors to occupy channels of PUs and SUs. There is an unlimited buffer. If mesh node $i$ want to send data to its neighbor $j$ and $A C_{i} \cap A C_{j} \neq \varnothing$, i selects the channel 
on which the overall transmission time of SU services is minimum. The overall transmission time of a service consists of the idle time (i.e., waiting time, $w$ ) from the moment that the service request initiates to the moment that it starts to transmit and the required time (i.e., service time, $s$ ) that the service starts to transmit on the channel until finished. The transmission of SU services may be broken off due to the appearance of PUs. It means that $s$ equals to $\mu_{\text {s }}$ plus the waiting time for to be resume the unfinished transmission after interruption.

According to PRP M/G/1 queuing theory in [10] [11], the time of the service of user $p$ waiting channel $q\left(q \in A C_{i} \cap A C_{j}\right)$ to be idle in the buffer is

$$
W(q)=\lambda_{p}^{q}\left(\mu_{p}^{(q)}\right)^{2}+\lambda_{s} \mu_{s}^{2} /\left(1-\rho_{p}^{(q)}\right)\left(1-\rho_{p}^{(q)}-\rho_{s}^{(q)}\right) .
$$

Where $\rho^{(q)}=\rho_{p}^{(q)}+\rho_{s}=\lambda_{p}^{(q)} \mu_{p}^{(q)}+\lambda_{s} \mu_{s} \leq 1$ represents the utilization of channel $q$.

Once PU appears, SU should vacate and enter into the buffer. Not until the channel becomes idle again will the unfinished transmission be resumed. So the total service time of the service of user $p$ on the channel $q\left(q \in A C_{i} \cap A C_{j}\right)$ is

$$
S(q)=\mu_{s}+P / Q \lambda_{p}^{(q)} \mu_{s} \mu_{p}^{(q)} / 1-\lambda_{p}^{(q)} \mu_{p}^{(q)} .
$$

Where $P / Q$ indicates that the service requests of PU select their operating channel equally.

Therefore, the per-hop spectrum decision metric, i.e. the overall transmission time of the service on channel $q\left(q \in A C_{i} \cap A C_{j}\right)$ is

$$
T(q)=W(q)+S(q)
$$

\section{CROSS-LAYER SCHEME BETWEEN SPECTRUM DECISION AND END-TO-END ROUTING}

The end-to-end performance of multi-hop wireless network can not be reflected by per-hop spectrum decision. Here, end-to-end cumulative delay is regarded as an indicator to of the network performance in CogWMN.

Assume that the channels on both sides of a relay node $r$ on the path from source to destination are different, like $c 1$ and $c 2$. At this time channel switch occurs, increasing end-to-end delay assuredly. It has been pointed out in [12] the delay caused by channel switch relates to the frequency distance of the two channels on the spectrum, formulated as:

$$
D_{c 1, c 2}=\beta \cdot\left|f_{c 1}-f_{c 2}\right|
$$

Where, $f_{c 1}$ and $f_{c 2}$ are the corresponding centre frequency of $c 1$ and $c 2 . \beta=1 \mathrm{~ms} / 10 \mathrm{MHz}$

Suppose that there are $x$ hops on the end-to-end route $l$. The metric for end-to-end route is defined as the end-to-end cumulative delay as follows:

$$
\operatorname{Metric}(l)=\sum_{y=1}^{x-1}\left(T^{\left(c_{y}\right)}+D_{c_{y}, c_{y+1}^{\prime}}\right)+T^{\left(c_{x}\right)}
$$

Here, Metric $(l)$ is the metric for route $l . T^{\left(c_{y}\right)}$ is the total transmission time of the service using channel $c$ on hop $y$. $D_{c_{y}, c_{y+1}^{\prime}}$ is the delay of channel switch from channel $c$ on hop $y$ to channel $c^{\prime}$ on hop $y+1$.

\section{PERFormance EVAlUation}

Nodes in CogWMN are distributed randomly in a $500 \mathrm{~m} \times 500 \mathrm{~m} \times 500 \mathrm{~m}$ space region. Each GERAN user is assigned a fixed channel and enjoys preemptive priority access. The bandwidth of each channel is $200 \mathrm{KHz}$. The transmission power of all the mesh nodes is set to $20 \mathrm{dBm}$. The carrier frequency is $900 \mathrm{MHz}$. SU services intelligently access to spectrum opportunities based on FCFS (First Come, First Service) scheduling policy. All the results following are derived from the average value of 1000-times Monte Carlo simulation.

\section{A. Per-hop Spectrum Decision}

Assuming that there are 8 PUs and the number of channels is $8 .{ }^{\mu_{s}}$ is $15 \mathrm{~s}$ and ${ }^{\lambda_{p}}$ is an arbitrary value between $0.01 / \mathrm{s}$ and $0.02 / \mathrm{s}$ (i.e. 36 72 average data calls per hour), $\mu_{p}$ between 10s and 20s. Select a couple of neighbors randomly to study the algorithms for per-hop spectrum decision and compare the network performance employing the proposed and the random decision algorithm. From Fig. 1 , our proposed algorithm works much better in terms of the overall transmission time of SU services. For instance, when the arrival rate of SU services $\lambda_{s}$ equals to $0.03 / \mathrm{s}$, the overall transmission time is reduced by $30 \%$ using our proposed algorithm nearly instead of the random decision algorithm. Furthermore, the overall transmission time increases with ${ }^{\lambda_{s}}$, which is because the increase in ${ }^{\lambda_{s}}$ brings the increase of SU services in the buffer, and thus the increase of waiting time.

While ${ }^{\lambda_{s}}$ equals to $0.02 / \mathrm{s}$, the differences on the overall transmission time of SU services of the two per-hop spectrum decision algorithms are described in Fig. 2. As can be seen, our proposed algorithm is a better choice for perhop channel selection. The overall transmission time increases with the increase in the number of mesh nodes because of the corresponding increase in the number of service requests. But for random method, there are no rules to follow, due to the randomness of channel selection.

\section{B. End-to-end Routing Selection}

Select a mesh node as the source and another as the destination randomly, and other parameters remain the same as above. The differences on end-to-end cumulative delay between our proposed cross-layer strategy and minimum hops based random channel selection are shown in Fig. 3 with respect to the number of mesh nodes, and Fig. 4 licensed channels. Here, the process of the latter includes a route selection using Dijkstra algorithm and each per-hop channel selection using random decision algorithm. The curve and bar graph proves that our proposed cross-layer scheme performs better on end-to-end cumulative delay.

\section{CONCLUSIONS}

Firstly, a per-hop spectrum decision algorithm has been proposed in this paper. In this algorithm, cognitive mesh nodes sense the licensed spectrum of GERAN system; calculate the available channel set of each per-hop link; 
analyze the quality of each available channel; select and access a channel which brings the minimum overall transmission time of SU services. Secondly, we propose a cross-layer scheme between link-layer spectrum decision and network-layer routing, with the objective of minimizing the end-to-end cumulative delay. Finally, with respect to the random channel selection algorithm based on Dijsktra shortest path, the cross-layer scheme can decrease the network end-to-end cumulative delay and thus improves the network transmission performance.

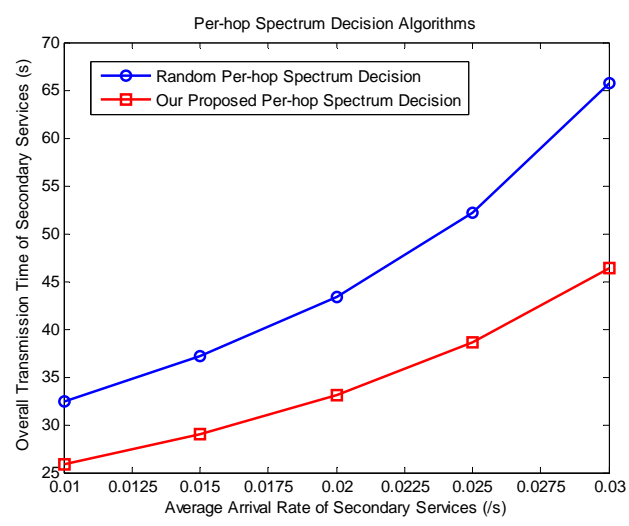

Figure 1. Effect of $\lambda_{\mathrm{s}}$ on overall transmission time

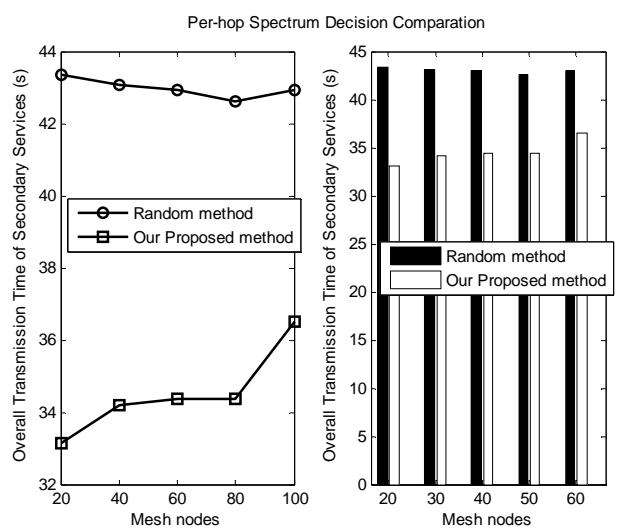

Figure 2. Effect of the number of mesh nodes on overall transmission time

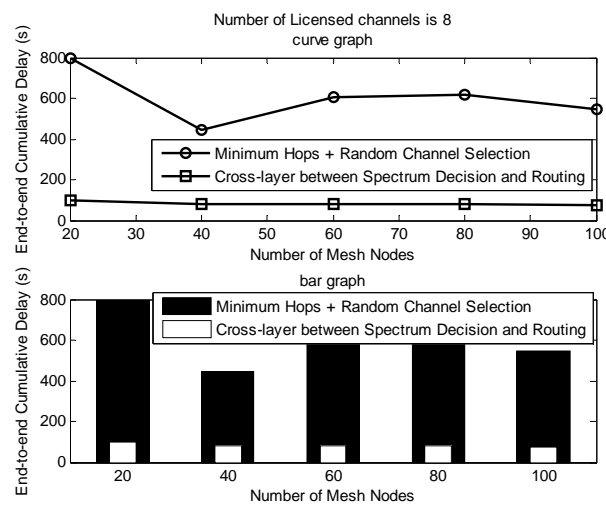

Figure 3. Effect of the number of mesh nodes on end-to-end delay

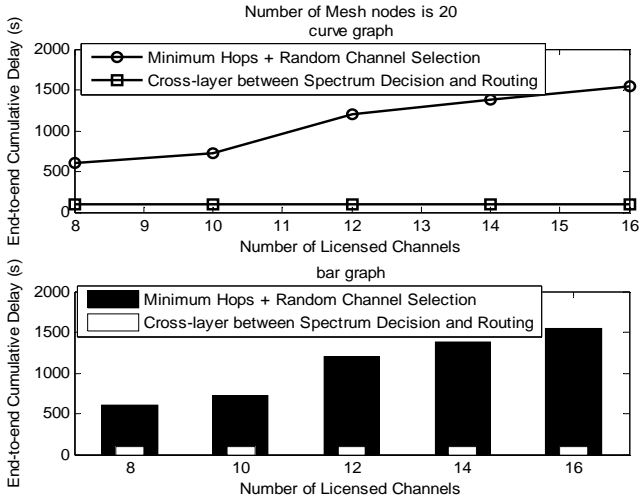

Figure 4. Effect of the number of licensed channels on end-to-end delay

\section{ACKNOWLEDGMENT}

This work was supported by the National Natural Science Foundation of China under Grant 61071086, the Project of Ministry of Transport of the People’s Republic of China under Grant 2012-319-813-270, Nantong Science and Technology Plan Project under Grant HS2011015, BK2011017, and Graduate Research and Innovation Plan Project of Nantong University under Grant YKC11058.

\section{REFERENCES}

[1] X. M. FANG, "Next-generation wireless internet technology: wireless mesh network,” Beijing: People's Posts and Telecommunication, 2005, pp.1-10.

[2] Q. ZHAO and B. M. SADLER, “A survey of dynamic spectrum access,” IEEE Signal Processing Magazine, vol. 24, no. 3, pp.79-89, May, 2007.

[3] FCC, "Establishment of an interference temperature metric to quantify and manage interference and to expand available unlicensed operation in certain fixed mobile and satellite frequency bands,” ET Docket No. 03-289, Notice of Inquiry and Proposed Rulemaking, December 2003.

[4] FCC, "Facilitating opportunities for flexible, efficient, and reliable spectrum use employing cognitive radio technologies," ET Docket No. 03322, Washington, 2003.

[5] T. CHEN, H. G. ZHANG, M. MATINMIKKO, and M.D. KATZ, "CogMesh: Cognitive wireless mesh networks," IEEE GLOBECOM Workshops, pp. 1-6, November-December 2008.

[6] N. BOUADBALLAH, B. ISHIBASHI, and A. R.BOUTAB, "Performance of cognitive radio-based wireless mesh networks," IEEE Transaction on Mobile Computing, vol. 10, no.1, pp.122-135, January, 2011.

[7] C. L. GUO, CH. Y. FENG, and ZH.M. ZENG, "Cognitive radio network technologies and applications,” Beijing: Electronic Industry, 2010, pp.151-166.

[8] E. HOSSAIN and V. K. BHARGAVA, "Cognitive wireless communication networks,” Vancouver: Springer, 2007, pp.440.

[9] L. MARIN and L. GIUPPONI, "Performance evaluation of spectrum decision schemes for a cognitive Ad-Hoc network,” IEEE $19^{\text {th }}$ International Symposium on PIMRC, pp.1-5, September, 2008.

[10] C. H. NG and B. H. SOONG, "Queuing modeling fundamentals: with applications in communication networks,” 2nd ed., US: Wiley, 2008.

[11] Y. YANG, G. A. ZHANG, and Y. CH. JI, "Impartial spectrum decision under interference temperature model in cognitive wireless mesh networks,” INCoS, pp.566-570, September, 2012.

[12] S. KRISHNAMURTHY, M. THOPPIAN, S. VENKATESAN, and R. PRAKASH, "Control channel based MAC-layer configuration, routing, and situation awareness for cognitive radio network,” IEEE MILCOM, pp.455460, October, 2005 